\title{
A multiscale approach to estimating topographically correlated propagation delays in radar interferograms
}

\section{Yu-nung Nina Lin}

Division of Geological and Planetary Sciences, California Institute of Tecbnology, MC100-23, 1200 East California Boulevard, Pasadena, California 91125, USA (ninalin@caltech.edu)

\section{Mark Simons}

Division of Geological and Planetary Sciences, California Institute of Technology, MC252-21, 1200 East California Boulevard, Pasadena, California 91125, USA

\section{Eric A. Hetland}

Department of Geological Sciences, University of Michigan, 2534 C. C. Little Building, 1100 North University Avenue, Ann Arbor, Michigan 48109, USA

\section{Pablo Muse}

Department of Signal and Image Processing, IIE, Facultad de Ingenieria, Universidad de la Republica, 11300

Montevideo, Uruguay

\section{Christopher DiCaprio}

Division of Geological and Planetary Sciences, California Institute of Technology, MC252-21, 1200 East California Boulevard, Pasadena, California 91125, USA

Now at ExxonMobil Upstream Research Co., Houston, Texas 77098, USA

[1] When targeting small amplitude surface deformation, using repeat orbit Interferometric Synthetic Aperture Radar (InSAR) observations can be plagued by propagation delays, some of which correlate with topographic variations. These topographically-correlated delays result from temporal variations in vertical stratification of the troposphere. An approximate model assuming a linear relationship between topography and interferometric phase has been used to correct observations with success in a few studies. Here, we present a robust approach to estimating the transfer function, $K$, between topography and phase that is relatively insensitive to confounding processes (earthquake deformation, phase ramps from orbital errors, tidal loading, etc.). Our approach takes advantage of a multiscale perspective by using a band-pass decomposition of both topography and observed phase. This decomposition into several spatial scales allows us to determine the bands wherein correlation between topography and phase is significant and stable. When possible, our approach also takes advantage of any inherent redundancy provided by multiple interferograms constructed with common scenes. We define a unique set of component time intervals for a given suite of interferometric pairs. We estimate an internally consistent transfer function for each component time interval, which can then be recombined to correct any arbitrary interferometric pair. We demonstrate our approach on a synthetic example and on data from two locations: Long Valley Caldera, California, which experienced prolonged periods of surface deformation from pressurization of a deep magma chamber, and one coseismic interferogram from the $2007 \mathrm{Mw} 7.8$ Tocapilla earthquake in northern Chile. In both examples, the corrected interferograms show improvements in regions of high relief, independent of whether or not we pre-correct the data for a source model. We believe that most of the remaining signals are predominately due to heterogeneous water vapor distribution that requires more sophisticated correction methods than those described here. 
Components: 7400 words, 12 figures, 1 table.

Keywords: atmospheric delay; multiscale analysis; InSAR; Long Valley Caldera; Tocopilla earthquake.

Index Terms: 1241 Geodesy and Gravity: Satellite geodesy: technical issues (6994, 7969); 1240 Geodesy and Gravity: Satellite geodesy: results $(6929,7215,7230,7240)$.

Received 14 May 2010; Revised 15 July 2010; Accepted 26 July 2010; Published 2 September 2010.

Lin, Y. N., M. Simons, E. A. Hetland, P. Muse, and C. DiCaprio (2010), A multiscale approach to estimating topographically correlated propagation delays in radar interferograms, Geochem. Geophys. Geosyst., 11, Q09002,

doi:10.1029/2010GC003228.

\section{Introduction}

[2] Interferometric Synthetic Aperture Radar (InSAR) images are widely used in the analysis of tectonic deformation, magmatic activity, flow of glaciers, and other surface deformation processes (for reviews, see Massonnet et al. [1994], Burgmann et al. [2000], Hanssen [2001], and Simons and Rosen [2007]). These observations are frequently plagued by spatially heterogeneous propagation delays between the radar platform and the ground. Propagation delays have three major sources: wet delays, hydrostatic delays and ionosphere effects. For microwaves, the refractivity changes due to the dipole components of water vapor, also known as "wet delays", contribute the most to the neutral atmospheric propagational delays [Bevis et al., 1992]. Recent study also shows that hydrostatic delays, which depends on the pressure to temperature ratio, varies significantly at low elevation and cannot be neglected [Doin et al., 2009]. Ionospheric effects result from spatio-temporal variations in ionospheric electron density. These effects are in general more obvious in the higher latitude and in the L-band SAR data than the C-band SAR, due to the dispersive nature of the atmospheric medium [Gray et al., 2000]. Wet delays and hydrostatic delays may strongly obscure tectonic signals when the signal amplitude is small as is frequently the case with interseismic deformation. For example, in two-pass interferometry, a $20 \%$ change in humidity may result in $10 \mathrm{~cm}$ of deformation error [Zebker et al., 1997], thereby compromising the effectiveness for InSAR to detect $\mathrm{mm}$-to-cm scale deformation. Wet delays and hydrostatic delays are non-dispersive and therefore the multiwavelength approach generally used to correct for GPS ionospheric biases cannot be applied to tackle this problem [Zebker et al., 1997].

[3] One way to mitigate the tropospheric delay problem is to average $\mathrm{N}$-independent interferograms, since the neutral atmospheric signals are uncorrelated over timescales longer than 1-day [Zebker et al., 1997; Emardson et al., 2003]. This simple technique can reduce the variance of atmospheric errors by a factor of $\sqrt{N}$. The averaging method is practical and effective when trying to estimate secular rates from a large number of interferometric pairs. However, if time-dependent deformation is expected or only a few interferograms are available, the stacking approach is no longer useful. One way to mitigate the tropospheric delays in a time series is to apply filtering techniques, such as the temporal plus spatial filters suggested by Berardino et al. [2002]. The choice of filter in this approach is subjective, leading to the concern of smoothing out signals over the same time scales as the noise.

[4] Other studies propose more complicated but direct methods for estimating and removing the effects of wet delays. Proposed methods include use of GPS data [Onn and Zebker, 2006] and radiometric data to produce zenith path delay difference maps for InSAR atmospheric correction, for instance using either MODIS (Moderate Resolution Imaging Spectroradiometer) [Li et al., 2005, 2006a], or MERIS (Medium Resolution Imaging Spectrometer, for ENVISAT system only) [ $\mathrm{Li}$ et al., 2006b, 2006c]. Other approaches use weather models together with radiometric data to generate an instant water vapor map, such as MERIS with MM5 (Mesoscale Meteorological Model) [Puysségur et al., 2007], or use a weather model only to predict atmospheric delays [Foster et al., 2006]. These imagery-based or model-based approaches may provide estimates of water vapor distribution from independent data sources or models at the time the SAR image was acquired. However, imagery-based approaches have limited application for older SAR images. MODIS and MERIS was launched in 1999 and 2002, respectively. Radiometric systems require solar illumination, so they can not be used to correct for SAR images acquired at night. Some calibra- 
tion statement is also necessary for the user to accommodate the radiometric data to different study areas. GPS-based approaches are limited by the density and existence of GPS stations in some remote area. Moreover, the efficacy of modelingbased approaches are still debated, especially the extent to which they consistently reduce or add noise to interferometric observations.

[5] The approach proposed in this study, compared to the aforementioned methods, is relatively simple and not limited by the availability of other independent data. Our approach focuses on mitigating the effects of the time-variable vertically stratified component of the atmospheric delays, as described by Hanssen [2001]. Correction of the static component is relatively straightforward and efficient, and in some cases can be very effective. In a study of the Lake Mead area, Nevada, Cavalié et al. [2007] showed that static tropospheric delays can be estimated by analyzing the correlation between phase and topography. (To be more accurate, it is a correlation between phase change and topography, but for simplicity we use "phase" to refer to "phase change"). This method, however, does not always work well because in some cases phase does not seem linearly related to topography due to multiple tectonic/non-tectonic sources and confounding effects of delays due to turbulent atmospheric circulation.

[6] Our study proposes an improved method that is less sensitive to all these confounding factors. We use a multiscale approach to estimate variations in topographically-correlated propagation delays. This approach is based on the same assumption made by earlier works of a linear relationship between phase and topography. We first test our approach in a synthetic example, and validate that in regions where there is no strong turbulent mixing, this approach can serve as the first order correction. We then demonstrate our method with examples from the Long Valley Caldera in California, and northern Chile.

\section{A Multiscale Approach to Estimating Topographically Correlated Delays}

\subsection{Model}

[7] Correlation between range change and topography results from the variation of the refractivity of the atmosphere along the vertical due to changes in pressure $(\mathrm{P})$, temperature $(\mathrm{T})$, humidity and water vapor content in the lowermost atmosphere between two SAR acquisitions [Hanssen, 2001]. For a vertically stratified troposphere model, if elevation changes across the scene, propagation delays vary at different elevation with a rate increasing with water vapor content and $\mathrm{P} / \mathrm{T}$ ratio [Doin et al., 2009]. In contrast to the effect of turbulent mixing, this vertical stratification is considered static over a given area throughout a certain period of time. As the concentration of water vapor generally decreases exponentially with elevation, the theoretical delay curve is an exponential function of elevation [Delacourt et al., 1998]. In an interferogram subject to only static tropospheric delays, the signal is the difference between the delay curves for two individual SAR acquisitions. If we take a Taylor series expansion over the resultant exponential function, and ignore the second order and higher terms, we can derive a simple linear relation

$$
\Delta \phi=b+K h
$$

where $b$ is a bias term and $K$ is the transfer function between topography $(h)$ and phase $(\Delta \phi)$ [Cavalié et al., 2007]. This simple linear model works for most weather conditions, except for extraordinary cases such as inverted or non-monotonic tropospheric stratifications. The transfer function, $K$, is best determined by a global rather than a local linear regression, since the phase/elevation relationship may have local trade-offs with the deformation/elevation relationship [Cavalié et al., 2007]. This transfer function is therefore considered as a scene-wide property rather than a value that changes heterogeneously across the scene. However, global correlations may result in large uncertainties in the transfer function, making it difficult to define the linear relationship, thus emphasizing the need for a robust method to determining $K$.

\subsection{Estimation Approach}

[8] Our approach explicitly recognizes that various length scales, $\lambda$, should have different sensitivities to different sources of confounding noises. For example, very large $\lambda(>100 \mathrm{~km})$ may be more sensitive to other processes such as tidal loading [DiCaprio and Simons, 2008] or orbital error, whereas the smallest $\lambda(\leq 2 \mathrm{~km})$ may not be very sensitive to larger-scale tropospheric signals. Surface deformation resulting from tectonic, magmatic or glacial processes also has a rich scale-dependent spectrum. In the presence of all these confounding factors, given that $K$ is assumed to be a global 

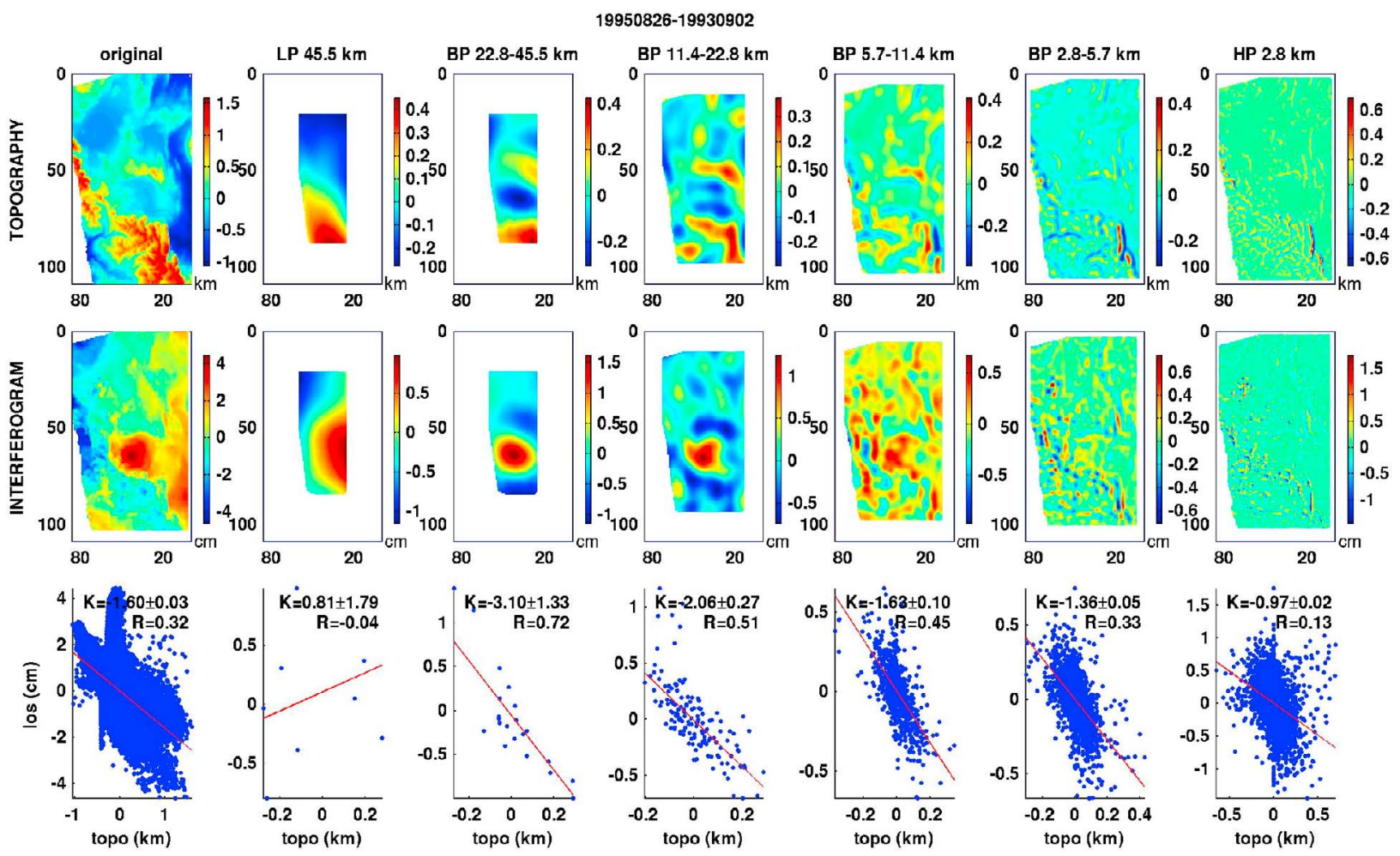

Figure 1. Original and decomposed (top) topography and (middle) interferogram. LP, BP and HP indicate low-pass, band-pass and high-pass respectively. The surrounding blank area in each channel results from omitting points along the scene boundaries to avoid edge effects when applying Gaussian filters. (bottom) The scatter plots of each decomposed band. The estimated value of $K$ with uncertainties and correlation coefficient $R$ for each channel are shown at the top right corners of the scatter plots. The final estimate of $K$ is $-1.53 \mathrm{~cm} / \mathrm{km}$ in this example, close to what is derived from full-interferogram correlation $(-1.60 \mathrm{~cm} / \mathrm{km})$. The interferograms are produced from ERS SAR images acquired on 1995/08/26 and 1993/09/02 (see Figure 3).

property, there should be a reasonable range of $\lambda$ in which the value of $K$ almost stays constant and is independent of $\lambda$. Therefore, we can take advantage of the multiscale perspective to robustly estimate a spatially constant $K$ which is relatively insensitive to confounding processes.

[9] To begin with, we decompose both topography and interferogram into different length scales (Figure 1). We generate band-passed images by applying a series of Gaussian filters with different spatial scales and taking the difference between two neighboring scales. We choose filter limits that scale with integer powers of two (in units of integer numbers of pixels). To properly represent the amount of information carried in each channel, we resample them according to Shannon-Nyquist sampling theorem. We use the resampled point sets from selected band-pass channels to estimate $K$.
[10] Band-pass (BP) components usually show clearer and more constant linear relationship between topography and phase than high-pass (HP) and low-pass components (LP). The high-pass and low-pass components are therefore excluded from the estimation of $K$. Next, we rewrite equation (1) as

$$
\Delta \phi\left(\lambda_{i}\right)=b_{\text {igram }}+K_{\text {igram }} h\left(\lambda_{i}\right)
$$

where $h\left(\lambda_{i}\right)$ and $\Delta \phi\left(\lambda_{i}\right)$ are the $i$ th band-passed components of $h$ and $\Delta \phi . b_{\text {igram }}$ and $K_{\text {igram }}$ denote the bias term and transfer function of each interferogram. When multiple interferograms are available for the same region we must estimate a consistent set of values for $K$. We do so by defining a unique set of component time intervals, $\Delta T$, for a suite of interferograms (Figure 2). Each $\Delta T$ has a corresponding $b_{\Delta T}$ and $K_{\Delta T}$, which represents the 


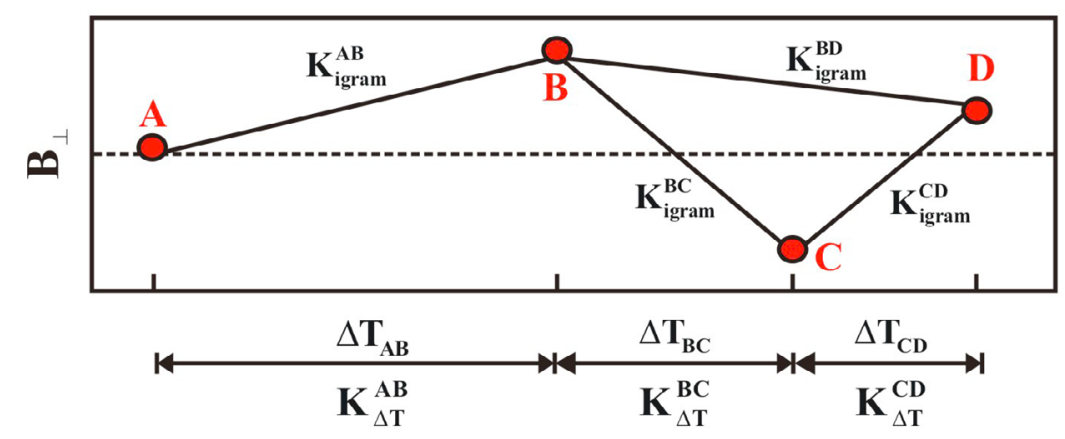

Figure 2. $K_{\Delta T}$ is formed by defining a unique set of component time interval $\Delta T$ based on the acquisition dates.

internally consistent $b$ and $K$ changes over this time interval. Next we construct the linear system sion, we estimate the standard error of $K_{\Delta T}\left(\sigma_{K_{\Delta T}}\right)$ using a bootstrapping technique. Given that InSAR where $h_{m}\left(\lambda_{n}\right)$ represents the $n$ selected decomposed bands of topography corresponding to $m$ interferograms, and $\Delta \phi_{m}\left(\lambda_{n}\right)$ represents the $n$ selected decomposed bands of $m$ interferograms, while $K_{\Delta T_{p}}$ and $b_{\Delta T_{p}}$ represent the transfer function and bias term for the $p$ th $\Delta T$. By solving this large system of equations, we can derive both the transfer function and bias term at the same time, making the whole system internally consistent.

[11] We assume that there exist minor outliers in the data due to unwrapping errors or other measurement or processing defects. Under this assumption, an outlier-resistant L1-norm regression is a better choice than least-squares regression. In practice, we use a convex optimization algorithm (available online as Matlab package cvx) (M. Grant and S. Boyd, CVX: Matlab software for disciplined convex programming, 2009, available at http://stanford.edu/boyd/cvx) for L1 regression to derive the best solution of $K_{\Delta T}$ [Boyd and Vandenberghe, 2004]. Since there is no analytical equation to define model errors of L1-norm regres- data are correlated in space, ideally we should include the full covariance matrix into our regressions. However, because we apply a multiscale decomposition by using a series of Gaussian filters, there is an issue of transforming covariance matrix into each band-pass channel. The details are beyond the scope of this study, so we ignore data covariance in our regressions. The reader should keep in mind that the standard errors of K may be larger than what we present if the full covariance is considered. Finally, we form the time series of $K_{T}$ by choosing an arbitrary origin (we use zero here) for the whole series and sequentially adding up all $K_{\Delta T}$. Once the time series of the transfer function $K_{T}$ is formed, it is easy to determine the $K_{\text {igram }}$ of an interferogram from any arbitrary pair of SAR scenes.

\subsection{Synthetic Test}

[12] Our synthetic test is based on the inflation event between 1997 and 1998 in the Long Valley Caldera, California (Figure 3) [Langbein, 2003]. 


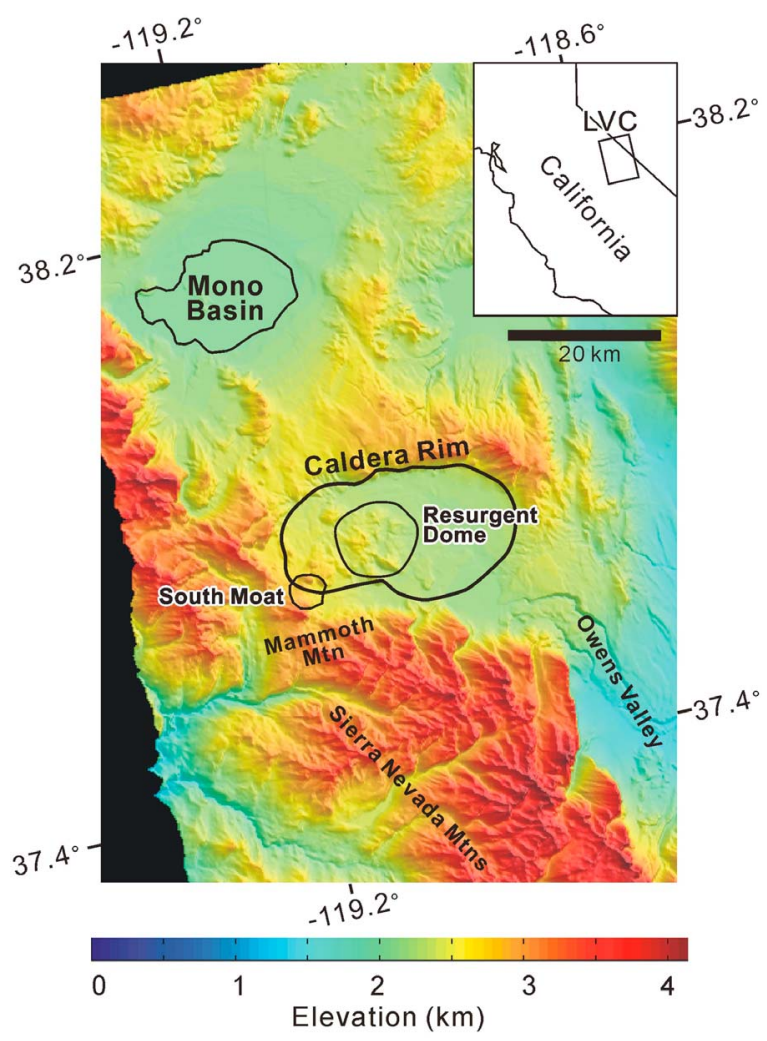

Figure 3. Reference map of Long Valley Caldera and the resurgent dome during the 1997-98 inflation episode. This map has the same extent as the data footprint of ERS track 485 used in this study.

The components considered in the synthetic interferogram include tectonic (magmatic inflation), static topographically-correlated delays, turbulent mixing and ramp signals. We construct the tectonic signals by using a point source of inflation in an elastic halfspace [Mogi, 1958]. We assume a source depth at $10.5 \mathrm{~km}$, consistent with Fialko et al. [2001]. As for turbulent mixing signals, several pre-determined noise covariance functions have been proposed, such as a power law or an exponential decay [Hanssen, 2001; Emardson et al., 2003; Lohman and Simons, 2005]. Here we chose the expression of Lohman and Simons [2005]

$$
\begin{aligned}
& C_{d}^{i j}=e^{-L_{i j} / L_{c}} \\
& n_{c}=v u^{1 / 2} n_{n}
\end{aligned}
$$

where $C_{d}^{i j}$ and $L_{i j}$ are the covariance and distance between the $i$ th and $j$ th points, $L_{c}$ is the scale distance, $n_{n}$ is uncorrelated noise, and $v$ and $u$ are the matrices of eigenvectors and eigenvalues of $C_{d}$, respectively. We assume a ramp that varies bili- nearly in space. The constructed ramp has a major gradient in the NW-SE direction, mimicking possible effects due to orbital error or horizontal water vapor gradients from north to south.

[13] There are three major parameters that we vary to see how they influence the estimate of $K$. The first one is the standard deviation $\sigma$ of noncorrelated noise $n_{n}$. The value is set to be between 0 to $5 \mathrm{~cm}$. The second parameter is the amplitude of the ramp. A small ramp has values between -0.5 to $0.5 \mathrm{~cm}$, close to the amplitude of tectonic signals. A large ramp has amplitude 10 times the small ramp. The last parameter is the characteristic length scale, $L_{c}$, of turbulent signals. $L_{c}$ is the distance over which the noise covariance decays by one fold. Estimates from real observations suggest a scale distance usually between 5 to $30 \mathrm{~km}$ [Lohman and Simons, 2005]. We test values of $5 \mathrm{~km}$, $15 \mathrm{~km}$ and $30 \mathrm{~km}$.

[14] Figure 4 shows one realization of our synthetic interferograms. We project all the components into the line-of-sight direction and combine them together. In total we generate 120 interferograms and retrieve $K$ values from each of them by using our multiscale approach. We then compare our results with the $K$ values derived from a full (not multiscale) interferogram-topography correlation, with either ramp retained or ramp removed (Figure 5). The results show that the multiscale approach gives a stable estimate of $K$ values regardless of noise strength. For the cases where $L_{c}=5 \mathrm{~km}, K$ values slightly deviate to higher values from the true $K$ as the noise $\sigma$ increases, but still within the error bars. In general, at greater noise levels, the multiscale approach tends to estimate transfer functions that are smaller than the real values. This tendency to under-estimate $K$ means that the multiscale approach is a more conservative method, so that in most cases it will under-correct rather than overcorrect the topographically-correlated tropospheric signals.

[15] We also considered the influence of phase ramps across the scene. $K$ values estimated from the ramp-retained full-interferogram correlation method are generally, but not always, better than those estimated from the ramp-removed fullinterferogram correlation method. A sophisticated ramp removal approach may allow one to avoid some intertwining signals, such as using the region outside the tectonically influenced zone to estimate the ramp [Cavalié et al., 2007]. In contrast to the full-interferogram approaches, the multiscale anal- 


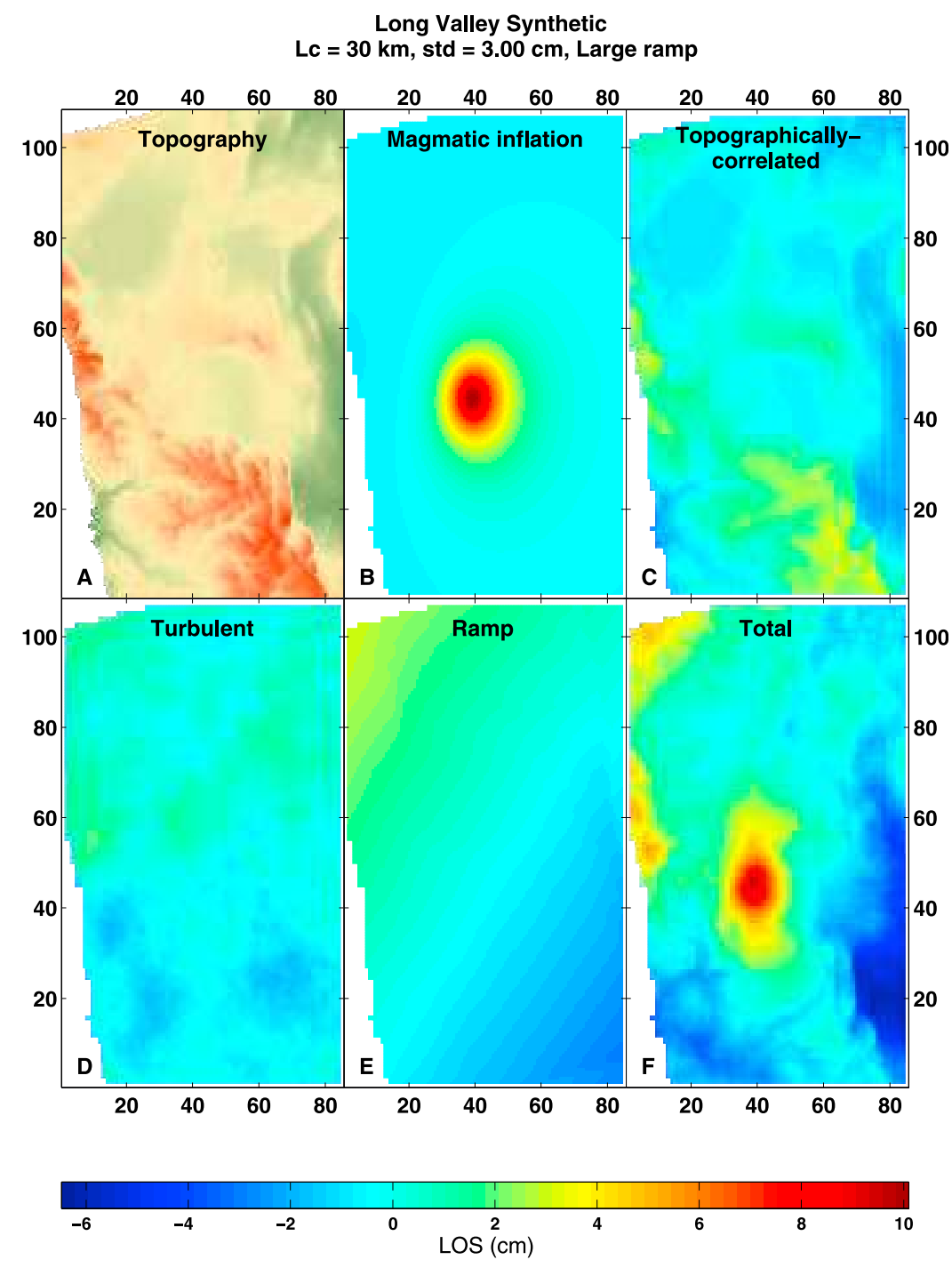

Figure 4. A schematic description of the construction of the synthetic interferometry. (a) The topography of Long Valley Caldera (location is the same as ERS track 485 in Figure 3), with maximum elevation (red color) up to $\sim 4 \mathrm{~km}$. We use the point source model of inflation $[\mathrm{Mogi}, 1958]$ to calculate the (b) line-of-sight surface displacement due to magmatic intrusion, and use topography to compute (c) topographically-correlated tropospheric delays. (d) Turbulent mixing signals and (e) small bilinear ramp are computed as described in the text. We project them to the line-of-sight direction and combine them to form the (f) final synthetic interferogram. In this example, $\sigma$ for $n_{n}$ is $3 \mathrm{~cm}$, and noise scale distance $L_{c}$ is $30 \mathrm{~km}$.

ysis is not sensitive to ramp magnitude and therefore no ramp removal is needed.

[16] $L_{c}$ also has some influence on the multiscale method. The retrieved $K$ values using the multiscale approach seem to be more stable at larger $L_{c}$. To explain this phenomenon, we compare the decomposed turbulent signals of different $L_{c}$ (Figure 6). At $L_{c}=5 \mathrm{~km}$, turbulent signals have more evenly spread amplitudes in all decomposed bands. As $L_{c}$ increases, turbulent signals become more concen- trated in the long-wavelength channels. Therefore in general, at large $L_{c}$, estimation of $K$ should be less influenced by turbulent mixing effects, but still depends on how the turbulent peaks and troughs randomly correlate to topography. In real cases, unfortunately, most turbulent signals are frequently related to topography. The user should hence keep in mind that the retrieved $K$ is likely to be degraded from the "true" $K$, with the level of deviation depending on the characteristic length scale and amplitude (standard deviation) of turbulent signals. 


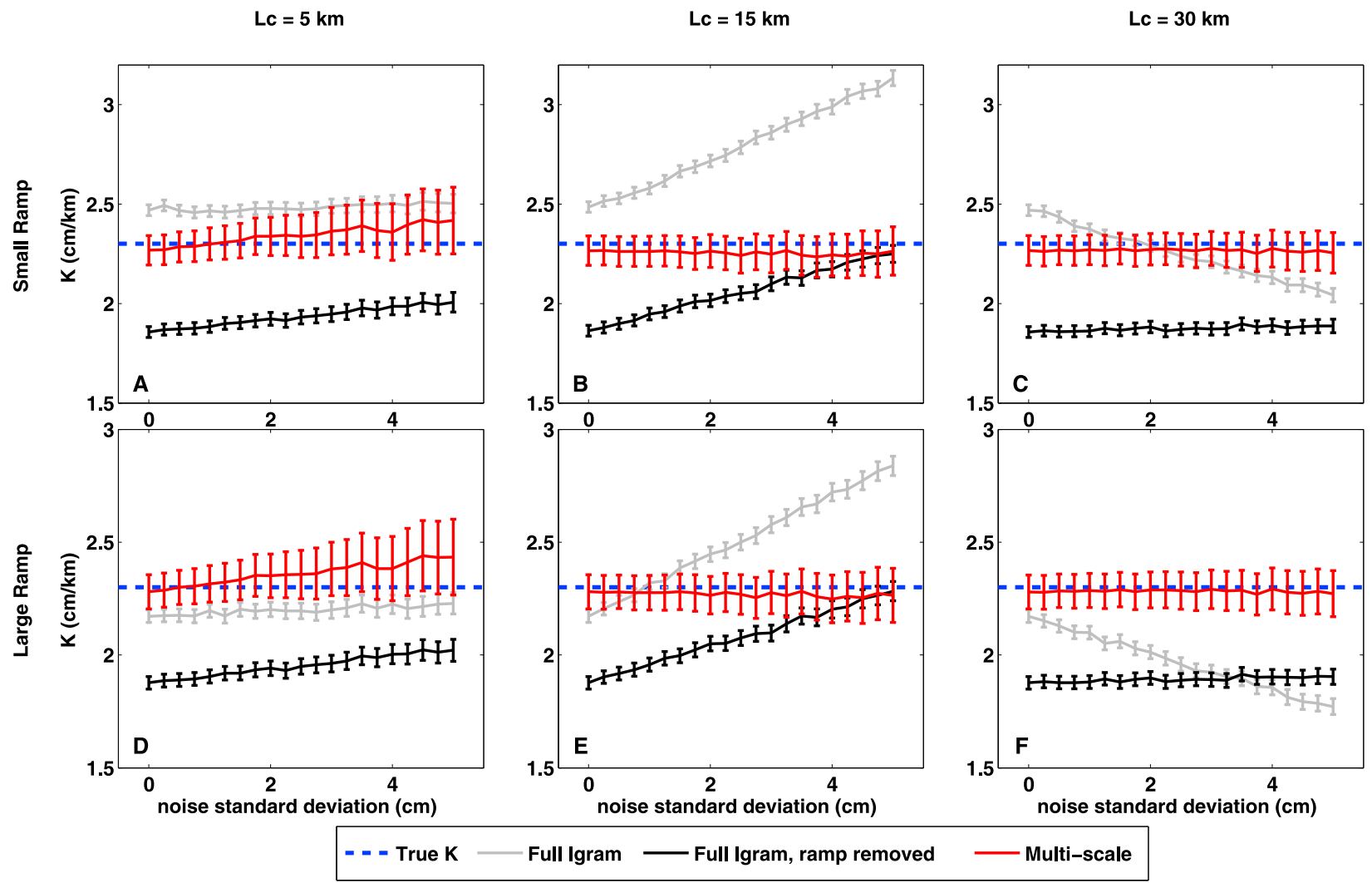

Figure 5. Comparison of $K$ values calculated by using full interferogram correlation (gray lines), full interferogram correlation with ramp removed (black lines) and multiscale analysis (red lines). There are 20 realizations of synthetic interferograms in each plot, with different levels of noise standard deviation. The input $K$ is $2.3 \mathrm{~cm} / \mathrm{km}$ (blue dashed lines). Among the three methods, the multiscale approach gives $K$ values that are most stable and closest to assigned $K$.

[17] To summarize the results from the synthetic test, we find that a multiscale approach provides a more robust way to estimate the transfer function, $K$. This approach is insensitive to phase ramps, and therefore can yield better estimates of $K$ when orbital error or long-wavelength deformation signals are present. Of course, as just alluded to, there may be a slight deviation of $K$ depending on the characteristic length scale and amplitude of turbulent signals.

\section{Correcting Real Interferograms}

[18] We test the multiscale approach in two study areas. Our first example is the 1997-98 magmatic inflation episode in Long Valley Caldera, California. This example presents a relatively simple tectonic source, combined with complicated atmospheric turbulent signals. We test the robustness of $K$ by removing the magmatic inflation signals from the interferograms. We also use this example to emphasize the stability of our algorithm in the presence of large-amplitude turbulent noise. Our second example, the 2007 Tocopilla earthquake (northern Chile), has "rich" tectonic signals that cover large area and wide range of wavelengths. We show how the $K$ values may vary with scene sizes and comment on whether it is reasonable to correct a large-size interferogram with a unique value of $K$. Since this study focuses on estimating topographically-correlated tropospheric signals, we do not discuss in detail the geophysical aspects of the two study cases.

\subsection{Long Valley Caldera}

[19] Long Valley Caldera has experience two phases of volcanic unrest since 1989. The first phase started rapidly in 1989, and slowly decayed through the early 1990s. The second phase started slowly in mid-1997, climaxed in late 1997, and returned to quiescence by mid-1998. During the second phase, it first showed an exponential growth 

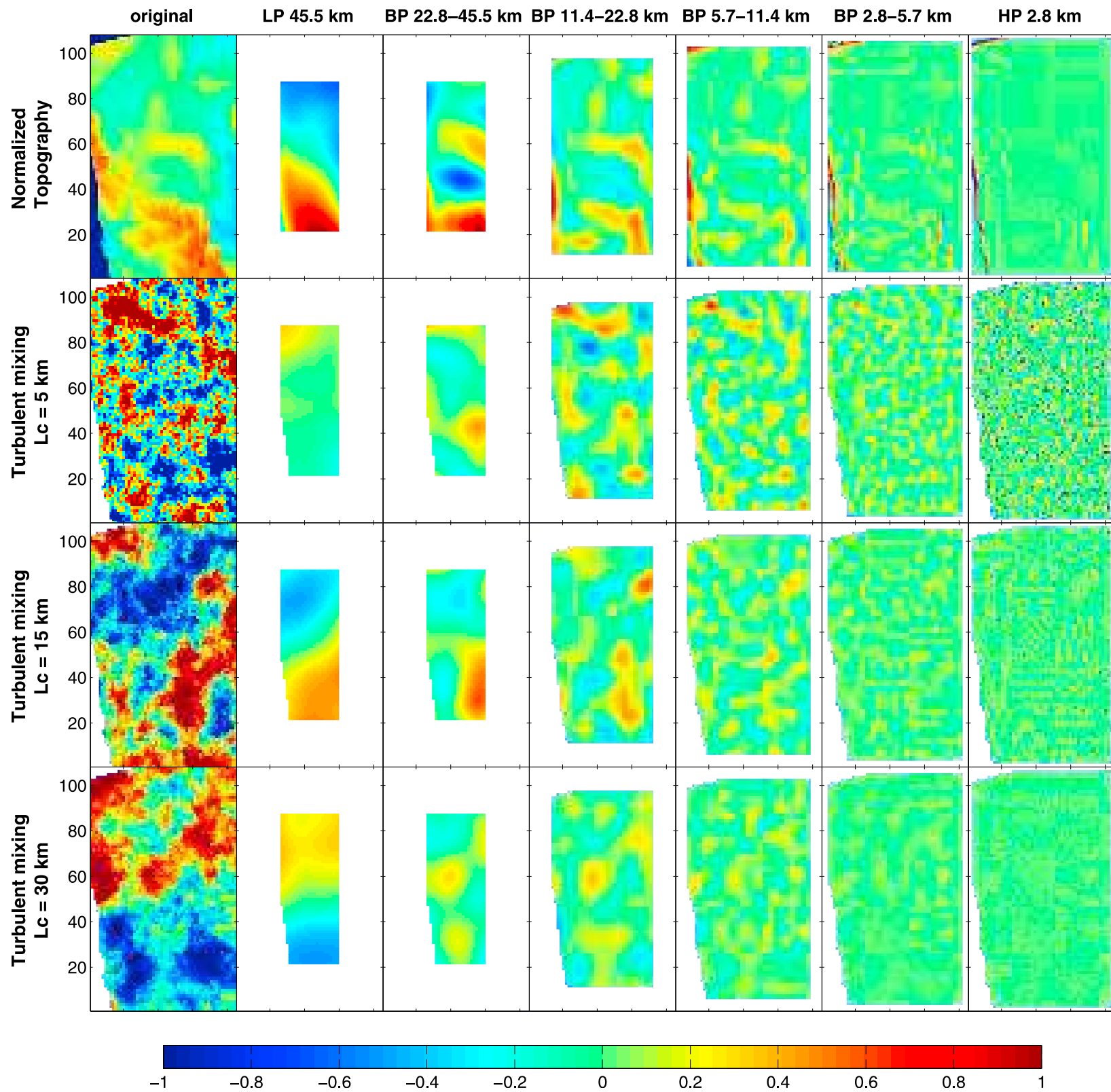

Figure 6. Comparison of decomposed turbulent signals with different scale distance $L_{c}$. Noise standard error is $2.5 \mathrm{~cm}$ in all cases. Notice that short-wavelength channels show higher amplitude at smaller $L_{c}$ than at larger $L_{c}$.

increase in mid-April, and an exponential growth decay in late November 1997, cumulating in $\sim 10 \mathrm{~cm}$ of uplift [Newman et al., 2001; Hill et al., 2003; Langbein, 2003].

[20] The only satellite that has acquisitions throughout the whole 1997-1998 Long Valley Caldera inflation episode is ERS. Unlike ENVISAT, ERS does not have any onboard device that measures water vapor content in real time. To remove troposphere-related delays, one may want to use
GPS data instead to model the water vapor distribution in that area. Unfortunately most of the available permanent GPS stations were established in 2000. In this case, our correction approach may serve as the best available tool to mitigate these static delays for older interferograms.

[21] We analyzed 65 interferograms based on 24 ERS acquisitions between 1992 and 2006. We tried to minimize the number of interferograms according to several criteria: (1) the length of the 


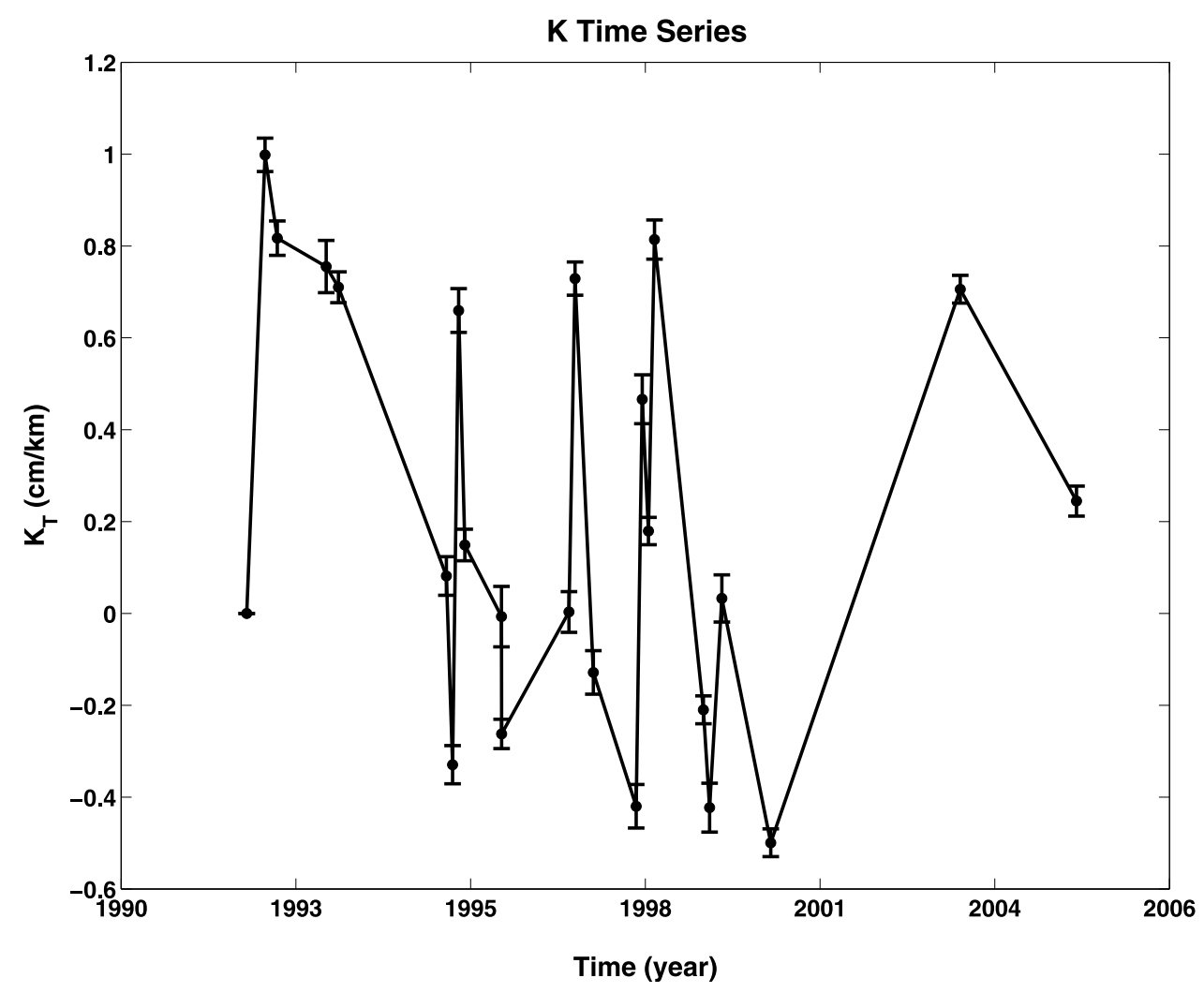

Figure 7. $K_{T}$ time series derived from multiscale approach. We arbitrarily set the first value in this $K_{T}$ time series as zero, and sequentially add up all $K_{\Delta T}$ values. The error bars here are shown with one standard error, derived by using bootstrapping technique.

perpendicular component of baseline $(\mathrm{B} \perp)$ should be less than $300 \mathrm{~m}$; (2) the date of acquisition should lie outside the winter season, according to snow precipitation record (Daily snow depth data of Rock Creek Lakes (RCK), California Data Exchange Center, Department of Water Resources, available at http://cdec.water.ca.gov/cgi-progs/ staMeta?station_id=RCK); and (3) the difference in Doppler frequency between two acquisitions must be less than $900 \mathrm{~Hz}$. The third criterion is particularly important, because the Doppler frequency starts to wander over a large range of values starting in 2001 [Meadows et al., 2007].

[22] We first carried out a multiscale decomposition of topography and interferograms with $720 \mathrm{~m}$ mean ground resolution. The length scales thus chosen, from low to high frequencies, are $>44.5,22.2-44.5$, 11.1-22.2, 5.6-11.1, 2.8-5.6 and $<2.8 \mathrm{~km}$. As we assume that smaller length scales $(\lambda \leq 2 \mathrm{~km})$ may not be sensitive enough to larger-scale tropospheric signals, there is no need to use higher data ground resolution, which also saves computation time. Of course, once $K$ is estimated, we can apply the correction to the full resolution interferogram. We then constructed and solved the linear system of equations (3). The time it takes to solve this linear system (65 interferograms) on an average PC is currently usually less than half an hour. We apply a bootstrapping technique [Tichelaar and Ruff, 1989] and derive the standard errors for $K_{\Delta T}$, with the average as $0.06 \mathrm{~cm} / \mathrm{km}$. Figure 7 shows the $K_{\Delta T}$ time series. Values of $K_{\text {igram }}$ estimated from $K_{\Delta T}$ are nearly identical to those estimated on an interferogram by interferogram basis, except for few outliers (Figure 8). After examining outlier interferograms individually, we found that these interferograms have larger areas with unwrappping errors. In this case our assumption that there are only minor unwrapping errors does not hold.

[23] We tested the sensitivity of our multiscale approach to confounding tectonic signals. We modeled the 1997-1998 inflation episode by using point inflation source [Mogi, 1958]. Source parameters are determined in the same way as in constructing synthetic interferograms. We assume that the source depth remains fixed during the whole inflation epi- 


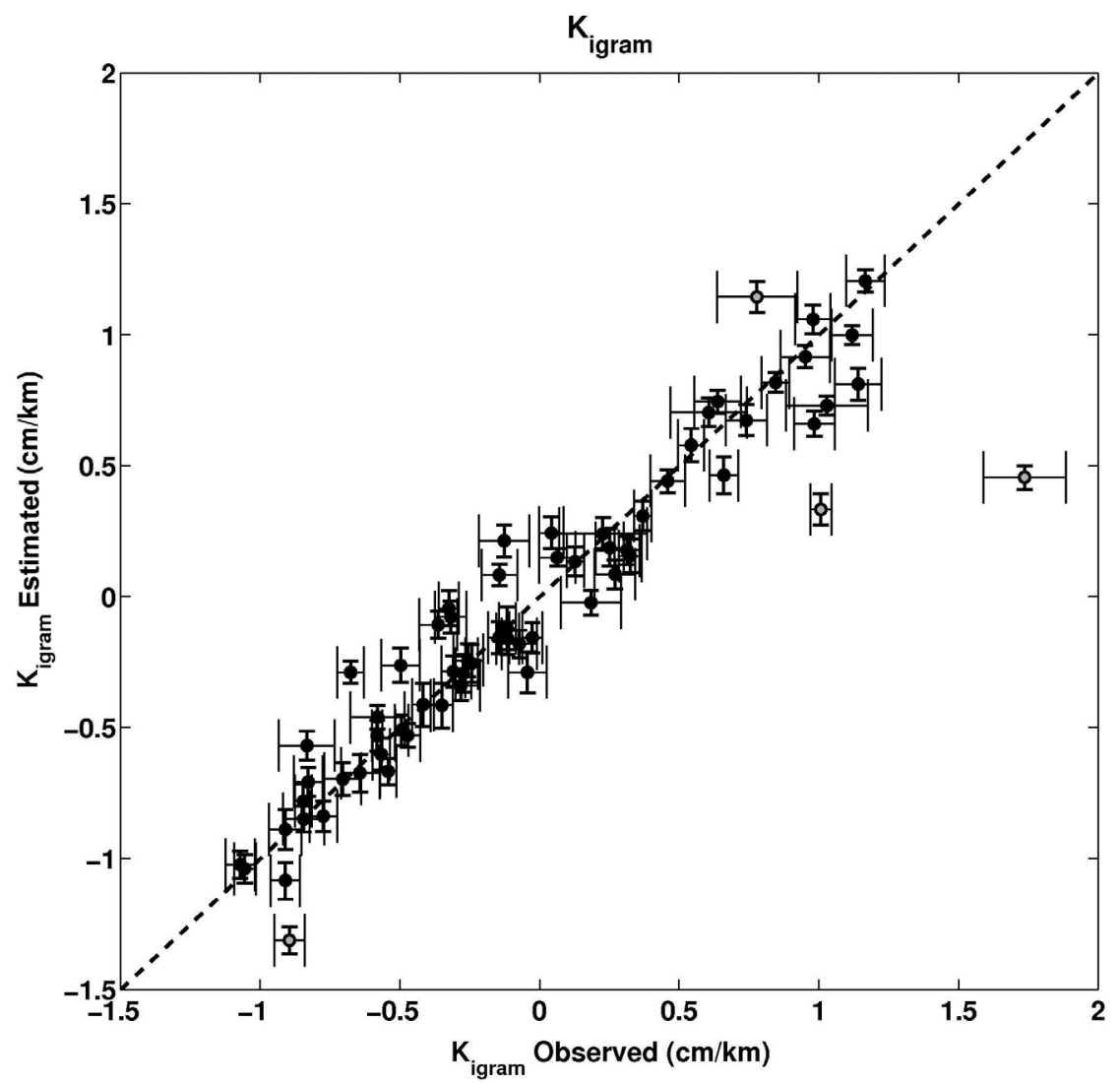

Figure 8. Comparison between observed $K_{\text {igram }}$, calculated directly from the phase-topography correlation of each interferogram as shown in equation (2), and estimated $K_{\text {igram }}$, derived from the $K$ time series. Outliers are shown in grey circles. These outliers result from larger unwrapping errors in the interferograms.

sode, with inflation volume as the only changing parameter. We then remove models from original interferograms, and carry out the multiscale decomposition and calculated the $K_{\text {igram }}$ value again. The $K_{\text {igram }}$ values thus derived are almost identical to the $K_{\text {igram }}$ values derived before model removal (Figure 9). This real case test proves that the estimate of $K$ by using multiscale approach is robust. The full-interferogram correlation approach, by contrast, does not seem to be stable after the inflation model is removed from the interferogram.

[24] This example also demonstrates significant influence of intermediate-wavelength turbulent disturbance, particularly near the center of the interferogram (Figure 9), where topography is not as high as Sierra Nevada. This turbulent disturbance is prominent in multiple interferograms, probably due to the water vapor brought by the northerly or southerly prevailing wind along Owens Valley [Zhong et al., 2008]. The phase-topography plot for each band-pass channel of this interferogram shows large scatter (Figure 10b), similar to what we derive from synthetic test when turbulent noise (with mid-to-large $L_{c}$ ) is high (Figure 10a). From what we have found through the synthetic test, our estimates of $K$ are more likely to under-correct than over-correct the interferograms.

\subsection{The 2007 Tocopilla Earthquake, Chile}

[25] The $\mathrm{M}_{w} 7.8$ Tocopilla earthquake occurred on 14 November 2007 (15:41 UTC) in northern Chile. The epicenter is located $25 \mathrm{~km}$ south of the town of Tocopilla and $150 \mathrm{~km}$ north-northeast of the city of Antofagasta (Figure 11) [Delouis et al., 2009]. The Global Centroid Moment Tensor (GCMT) catalog solution for the mainshock shows a centroid depth of $38 \mathrm{~km}$ and a focal mechanism of a low-angle nodal plane with reverse motion. The solution suggests that this event should be categorized as a subduction underthrusting earthquake occurring at the interface between the subducting Nazca plate and the overriding South American plate. The 


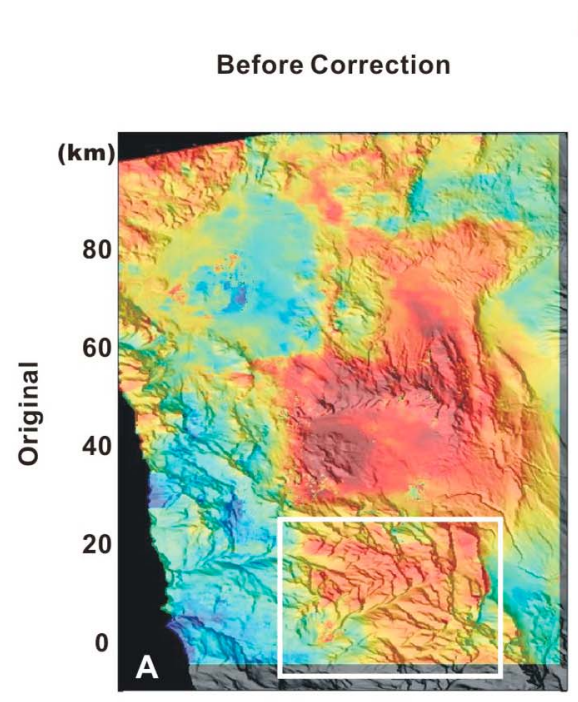

Long Valley, 1997/ 7/28 - 1995/ 8/26
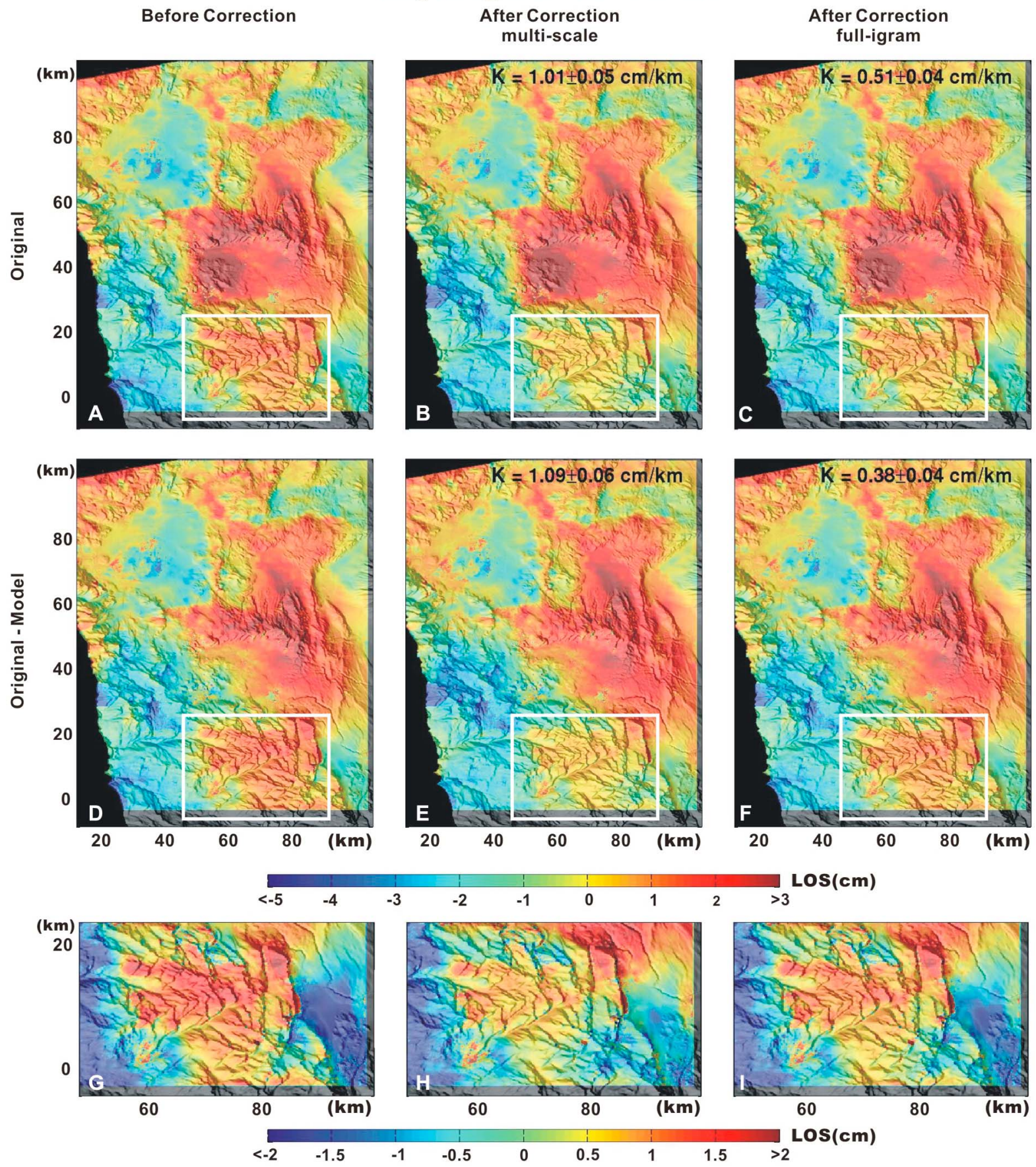

Figure 9. Comparison between the interferograms in Long Valley Caldera example before and after correction by using multiscale approach and full-interferogram correlation approach (ramp-retained). (a-c) The original interferograms before and after correction. ( $\mathrm{d}-\mathrm{f})$ The interferograms with inflation model subtracted before and after correction. The delays around the Sierra Nevada Mountains area (white rectangle) are properly removed after applying multiscale correction. (g-i) The enlarged plots of the area within the white rectangles in Figures 9a-9c. The large blob of noise near the center of the interferogram does not correlate well with topography and may mostly result from heterogeneous water vapor distribution in Owens Valley. 

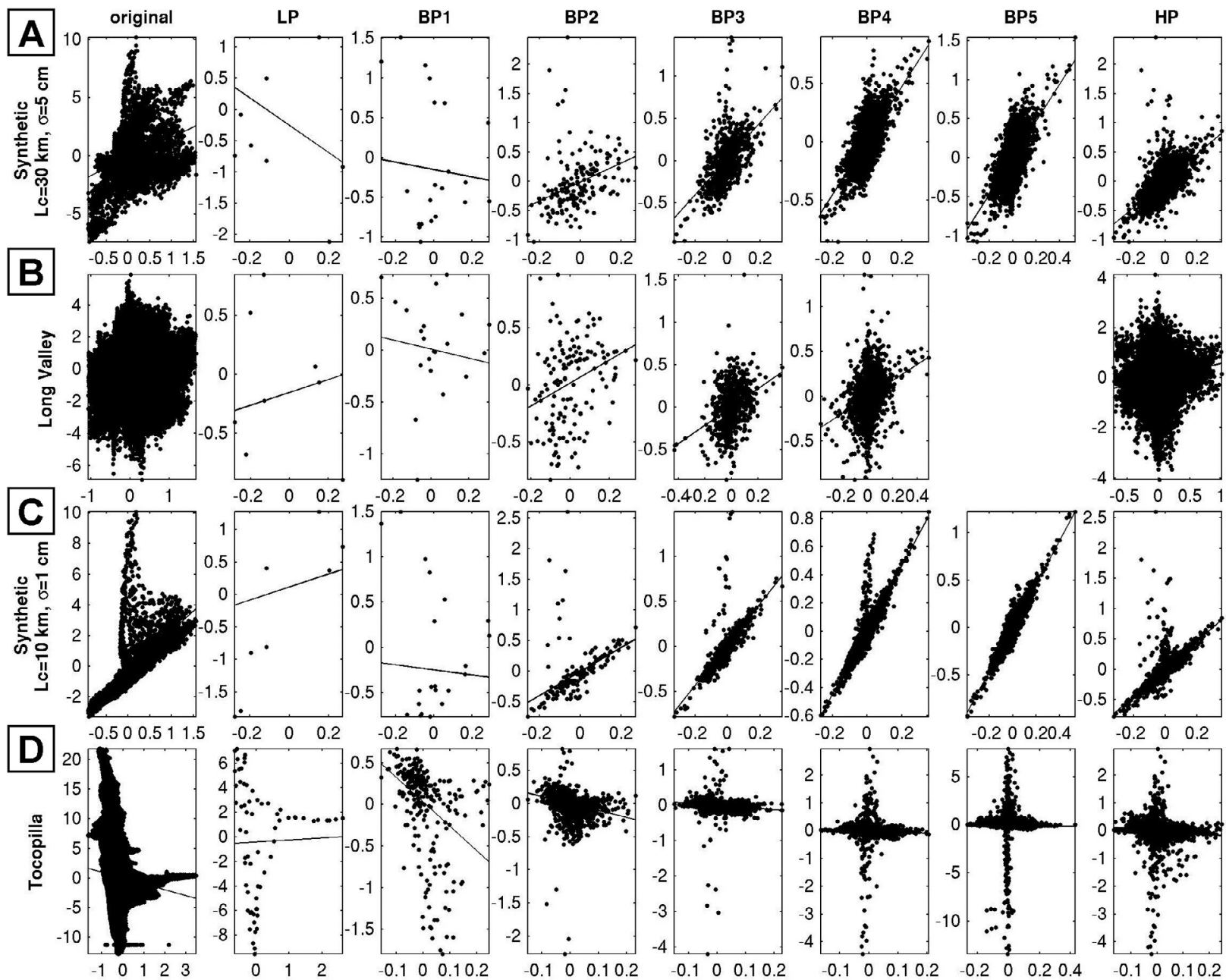

Figure 10. Scatter plots (phase vs. topography) between two real cases, Long Valley Caldera and Tocopilla, and two synthetic examples. This comparison verifies the high-amplitude and relatively low-amplitude turbulent signals in the Long Valley Caldera and Tocopilla interferograms, respectively. The high amplitude of turbulence in the Long Valley Caldera may suggest that the $K$ derived from multiscale approach may be smaller than "real" values, as shown by the synthetic test (Figure 5).

resultant tectonic signal therefore covers a large area (over $100 \mathrm{~km} \times 500 \mathrm{~km}$ ).

[26] We obtained data from ENVISAT ASAR descending track 96 acquired on April 9, 2007 and December 10, 2007 (Figure 11). We assume that the interferogram made from these two SAR scenes is dominated by coseismic deformation signals. We use the aftershock distribution to construct the fault plane, and use a finite-fault inversion routine [ $\mathrm{Ji}$ et al., 2002a, 2002b] to invert for the slip on the fault plane with constraints from both the interferogram measurements. We then remove the modeled displacement field from the interferogram. We estimate $K$ values with the model-retained and modelremoved interferograms, and find very close results:
$0.43 \pm 0.01$ and $0.48 \pm 0.01 \mathrm{~cm} / \mathrm{km}$ (Figure 12) These two values do not fall into each other's $95 \%$ confidence interval, but this is likely due to the fact that we did not consider the full covariance matrix in our calculation, which is computationally expensive but gives larger and more reasonable values for standard errors [Lohman and Simons, 2005]. After correcting the topographically-correlated tropospheric signals by using these two $K$ values, we clearly see that the phase gradient in the Andes (northern part of the interferogram) is reduced (Figures 12b and 12e). Compared with the multiscale correction result, the full-interferogram correlation removes even more of the gradient, but the derived $K$ value is not stable, and in some regions there exists the possibility of over-correction 


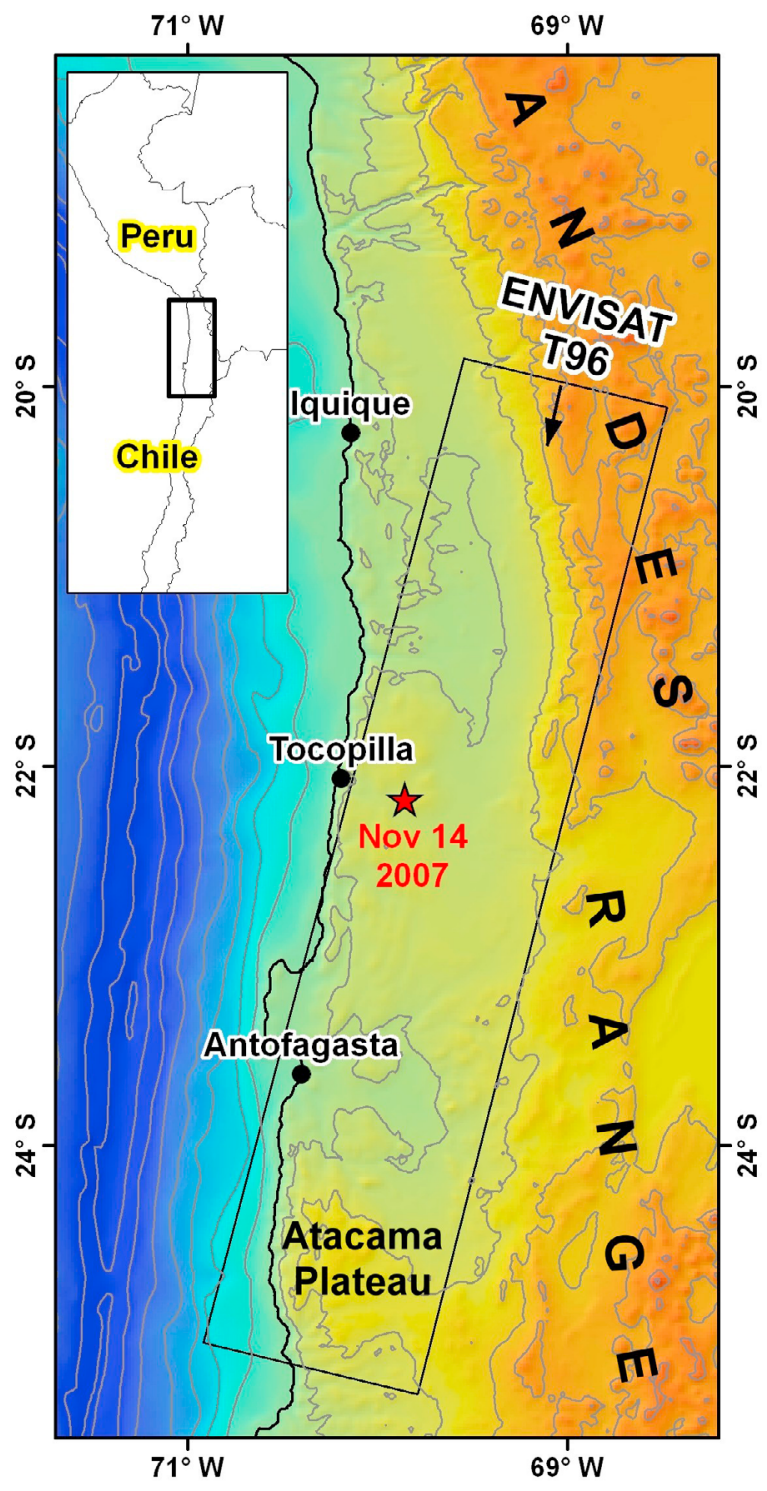

Figure 11. Reference map of the 2007 Tocopilla earthquake and the ENVISAT descending track 96. Contour interval is $1000 \mathrm{~m}$.

(Figure 12f, near the northeastern corner of the Andes). In the southernmost part of the interferogram near the Atacama Plateau, the phase increases after applying both corrections. It looks like on the original interferogram, there is positive phase-topography correlation, opposite to the trend in the Andes (Figure 12a). However, if we consider the phase change all the way from Antofagasta up to the Atacama Plateau, the phase decreases with elevation. Therefore, the $K$ values derived from both methods are self consistent within the whole interferogram, and the correction result should be valid.
[27] With an interferogram of such large scene size $(120 \times 590 \mathrm{~km})$, we should consider the validity of using a single value for $K$ to correct the whole interferogram. We test this idea by cropping the interferogram into various scene sizes and carrying out multiscale correction individually. The resultant $K$ values do not stay constant (Table 1). The values of $K$ derived for the sub-scenes using the multiscale approach do not vary nearly as much as do the values estimated by using the standard approach. Furthermore, the average of the $K$ values derived from each sub-scene is close to the $K$ value derived from the full-length interferogram by using the multiscale approach. Therefore for a given scene, the $K$ value represents the average condition of the vertically stratified troposphere in that given area. Treatment of a system in which $K$ varies slowly in space will be confounded by the effects of convective processes, and is thus not likely to be fruitful.

\section{Discussion and Conclusions}

[28] In the synthetic test, we show that the multiscale approach is insensitive to phase ramps. One important implication of this result is that we can apply this correction before baseline re-estimation without confounding the orbital phase ramp with topographically-correlated tropospheric signals. When re-estimating baseline model parameters from the unwrapped phase and a DEM, large-scale differential atmospheric artifacts will be aliased into the baseline estimate [Buckley et al., 2003]. Li et al. [2006a] showed that correcting the interferogram for atmospheric artifacts can effectively improve estimates of baseline parameters. When no satellite imagery-based or modeling-based correction method is available, incorporating a multiscale correction approach can reduce the long-wavelength topographically-correlated phase to a reasonable extent without over-estimating it, allowing more accurate baseline refinement.

[29] We demonstrate the robustness of the estimation of $K$ in two examples by using real observations. In our Long Valley Caldera example, the corrected interferograms still show strong delays near the caldera. This phenomenon is observed in multiple interferograms, probably due to the water vapor brought by the northerly or southerly prevailing winds in the Owens Valley. This dynamic signal has a strong amplitude $(\sim 3-5 \mathrm{~cm})$ and intermediate spatial wavelength $(\sim 20-40 \mathrm{~km}$; Figure 10). Despite the existence of such prominent 

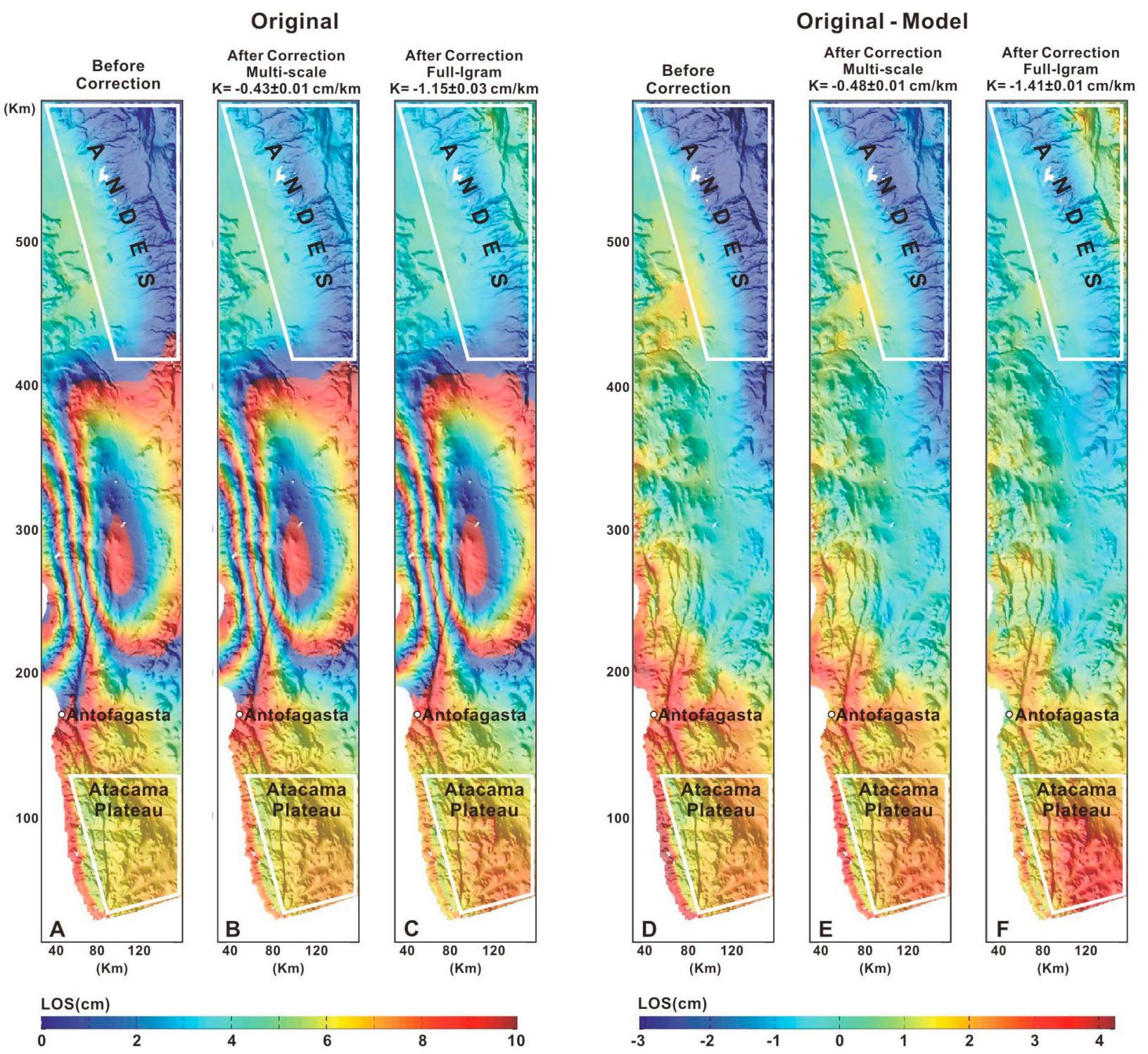

Figure 12. Comparison between the interferograms in the 2007 Tocopilla coseismic displacement example before and after correction by using multiscale approach. $(\mathrm{a}-\mathrm{c})$ The original interferograms before and after correction, wrapped by every $10-\mathrm{cm}$ displacement. $(\mathrm{d}-\mathrm{f})$ The model-removed interferograms before and after correction. Notice that the phase gradient around the Andes (white polygons) is reduced after correction. The scatter plots of each bandpass channel are shown in Figure 10d. Those plots indicate relatively small influence of turbulent signals in the Tocopilla example. See text for more discussion about the comparison of the multiscale and full-interferogram correlation methods, and the explanation of the correction result in the Atacama Plateau region.

turbulent mixing signals, out synthetic test shows that the multiscale approach still can provide with a robust and conservative estimate of $K$. In the Tocopilla example, the cropping test shows that $K$ value can vary with scene sizes. The $K$ value derived from the full-length interferogram by using multiscale approach represents an average of the vertically stratified troposphere in the given area, and therefore is a more conservative estimate of the transfer function. In conclusion, our multiscale correction should be considered as a fast and handy tool when nothing else is available, but it is not a panacea that can cure all challenges posed by tropospheric delays. 
Table 1. Variation of $K$ Values With Change of Scene Size in Tocopilla Example

\begin{tabular}{lcc}
\hline \multicolumn{1}{c}{ Scene Size $^{\mathrm{a}}$} & Multiscale & Full-Interferogram \\
\hline Full length & $-0.48 \pm 0.01$ & $-1.15 \pm 0.03$ \\
$1 / 2$ length $(291-580 \mathrm{~km})$ & $-0.46 \pm 0.02$ & $-0.60 \pm 0.14$ \\
$1 / 2$ length $(0-290 \mathrm{~km})$ & $-0.50 \pm 0.04$ & $-2.34 \pm 0.11$ \\
Average of two $1 / 2 \mathrm{scenes}$ & $-0.48 \pm 0.04$ & $-1.47 \pm 0.18$ \\
$1 / 4$ length $(436-580 \mathrm{~km})$ & $-0.35 \pm 0.02$ & $-1.07 \pm 0.01$ \\
$1 / 4$ length $(291-435 \mathrm{~km})$ & $-1.15 \pm 0.02$ & $-1.51 \pm 0.05$ \\
$1 / 4$ length $(146-290 \mathrm{~km})$ & $-0.11 \pm 0.04$ & $-19.83 \pm 0.19$ \\
$1 / 4$ length $(0-145 \mathrm{~km})$ & $-0.53 \pm 0.02$ & $-0.50 \pm 0.01$ \\
Average of four $1 / 4$ scenes & $-0.52 \pm 0.05$ & $-5.73 \pm 0.20$ \\
\hline
\end{tabular}

${ }^{\mathrm{a}}$ Full length: $120 \mathrm{~km} \times 580 \mathrm{~km}$; $1 / 2$ length: $120 \mathrm{~km} \times 290 \mathrm{~km}$; $1 / 4$ length: $120 \mathrm{~km} \times 145 \mathrm{~km}$.

\section{Acknowledgments}

[30] We would like to thank UNAVCO and GeoEarthScopes for supplying ERS images and European Space Agency (ESA) for supplying ENVISAT images through a category 1 project. We thank Sarah Minson at Caltech for providing the coseismic deformation model used in the Tocopilla earthquake example. We also thank Sebastien Leprince and Rowena Lohman for their valuable discussions and help in code development. This research is partially funded by NSF grant NNX09AD25G. This paper is Caltech Tectonic Observatory contribution 132 and Seismolab contribution 10044.

\section{References}

Berardino, P., G. Fornaro, R. Lanari, and E. Sansosti (2002), A new algorithm for surface deformation monitoring based on small baseline differential SAR interferograms, IEEE Trans. Geosci. Remote Sens., 40, 2375-2383.

Bevis, M., S. Businger, T. Herring, C. Rocken, R. Anthes, and R. Ware (1992), GPS meteorology: Remote sensing of atmospheric water vapour using the Global Positioning System, J. Geophys. Res., 97, 15,787-15,801.

Boyd, B., and L. Vandenberghe (2004), Convex Optimization, Cambridge Univ. Press, Cambridge, U. K.

Buckley, S., P. Rosen, S. Hensley, and B. Tapley (2003), Land subsidence in Houston, Texas, measured by radar interferometry and constrained by extensometers, J. Geophys. Res., 108(B11), 2542, doi:10.1029/2002JB001848.

Burgmann, R., P. Rosen, and E. Fielding (2000), Synthetic aperture radar interferometry to measure Earth's surface topography and its deformation, Annu. Rev. Earth Planet. Sci., 28, 169-209.

Cavalié, O., M.-P. Doin, C. Lasserre, and P. Briole (2007), Ground motion measurement in the Lake Mead area, Nevada, by differential synthetic aperture radar interferometry time series analysis: Probing the lithosphere rheological structure, J. Geophys. Res., 112, B03403, doi:10.1029/2006JB004344. Delacourt, C., P. Briole, and J. Achache (1998), Tropospheric corrections of SAR interferograms with strong topography: Application to Etna, Geophys. Res. Lett., 25, 2849-2852.

Delouis, B., M. Pardo, D. Legrand, and T. Monfret (2009), The Mw 7.7 Tocopilla earthquake of 14 November 2007 at the southern edge of the Northern Chile Seismic Gap:
Rupture in the deep part of the coupled plate interface, Bull. Seismol. Soc. Am., 99(1), 87-94, doi:10.1785/0120080192.

DiCaprio, C., and M. Simons (2008), The importance of ocean tidal load corrections for differential InSAR, Geophys. Res. Lett., 35, L22309, doi:10.1029/2008GL035806.

Doin, M. P., C. Lasserre, G. Peltzer, O. Cavalie, and C. Doubre (2009), Corrections of stratified tropospheric delays in SAR interferometry: Validation with global atmospheric models, J. Appl. Geophys., 69(1), 35-50, doi:10.1016/j.jappgeo. 2009.03.010.

Emardson, T., M. Simons, and F. Webb (2003), Neutral atmospheric delay in interferometric synthetic aperture radar applications: Statistical description and mitigation, J. Geophys. Res., 108(B5), 2231, doi:10.1029/2002JB001781.

Fialko, Y., M. Simons, and Y. Khazan (2001), Finite source modeling of magmatic unrest in Socorro, New Mexico, and Long Valley, California, Geophys. J. Int., 146, 191-200.

Foster, J., B. Brooks, T. Cherubini, C. Shacat, S. Businger, and C. Werner (2006), Mitigating atmospheric noise for InSAR using a high resolution weather model, Geophys. Res. Lett., 33, L16304, doi:10.1029/2006GL026781.

Gray, A. L., K. E. Mattar, and G. Sofko (2000), Influence of ionospheric electron density fluctuations on satellite radar interferometry, Geophys. Res. Lett., 27(10), 1451-1454.

Hanssen, R. F. (2001), Radar Interferometry, Data Interpretation and Error Analysis, Springer, New York.

Hill, D., J. Langbein, and S. Prejean (2003), Relations between seismicity and deformation during unrest in Long Valley Caldera, California, from 1995 through 1999, J. Volcanol. Geotherm. Res., 127, 175-193, doi:10.1016/S0377-0273 (03)00169-0.

Ji, C., D. Wald, and D. Helmberger (2002a), Source description of the 1999 Hector Mine, California, earthquake, part I: Wavelet domain inversion theory and resolution analysis, Bull. Seismol. Soc. Am., 92(4), 1192-1207.

Ji, C., D. Wald, and D. Helmberger (2002b), Source description of the 1999 Hector Mine, California, earthquake, part II: Complexity of slip history, Bull. Seismol. Soc. Am., 92(4), 1208-1226.

Langbein, J. (2003), Deformation of the Long Valley caldera, California: Inferences from measurements from 1988 to 2001, J. Volcanol. Geotherm. Res., 127, 247-267, doi:10.1016/S0377-0273(03)00172-0.

Li, Z., J.-P. Muller, and P. Cross (2005), Interferometric synthetic aperture radar (InSAR) atmospheric correction: GPS, Moderate Resolution Imaging Spectroradiometer (MODIS), and InSAR integration, J. Geophys. Res., 110, B03410, doi:10.1029/2004JB003446.

Li, Z., E. Fielding, P. Cross, and J.-P. Muller (2006a), Interferometric synthetic aperture radar atmospheric correction: GPS topography-dependent turbulence model, J. Geophys. Res., 111, B02404, doi:10.1029/2005JB003711.

Li, Z., E. Fielding, P. Cross, and J.-P. Muller (2006b), Interferometric synthetic aperture radar atmospheric correction: Medium Resolution Imaging Spectrometer and Advanced Synthetic Aperture Radar integration, Geophys. Res. Lett., 33, L06816, doi:10.1029/2005GL025299.

Li, Z., J.-P. Muller, P. Cross, P. Albert, J. Fischer, and R. Bennartz (2006c), Assessment of the potential of MERIS near-infrared water vapour products to correct ASAR interferometric measurements, Int. J. Remote Sens., 27, 349-365, doi:10.1080/01431160500307342.

Lohman, R., and M. Simons (2005), Some thoughts on the use of InSAR data to constrain models of surface deformation: 
Noise structure and data downsampling, Geochem. Geophys. Geosyst., 6, Q01007, doi:10.1029/2004GC000841.

Massonnet, D., K. Feigl, M. Rossi, and F. Adragna (1994), Radar interferometric mapping of deformation in the year after the Landers earthquake, Nature, 369(6477), 227-230.

Meadows, P., B. Rosich, A. Pilgrim, and M. Tranfaglia (2007), ERS-2 SAR performance and product evolution, in Proceedings of the Envisat Symposium 2007, Montreux, Switzerland, Eur. Space Agency Spec. Publ., ESA SP-636, 23-27.

Mogi, K. (1958), Relations between the eruption of various volcanoes and the deformation of the ground surface around them, Bull. Earthquake Res. Inst., 36, 99-143.

Newman, A., T. Dixon, G. Ofoegbu, and J. Dixon (2001), Geodetic and seismic constrains on recent activity at Long Valley Caldera, California: Evidence for viscoelastic rheology, J. Volcanol. Geotherm. Res., 105, 183-206, doi:10.1016/ S0377-0273(00)00255-9.

Onn, F., and H. A. Zebker (2006), Correction for interferometric synthetic aperture radar atmospheric phase artifacts using time series of zenith wet delay observations from a GPS network, J. Geophys. Res., 111, B09102, doi:10.1029/ 2005JB004012.
Puysségur, B., R. Michel, and J.-P. Avouac (2007), Tropospheric phase delay in interferometric synthetic aperture radar estimated from meteorological model and multispectral imagery, J. Geophys. Res., 112, B05419, doi:10.1029/ 2006JB004352.

Simons, M., and P. Rosen (2007), Interferometric synthetic aperture radar geodes, in Treatise on Geophysics, vol. 3, Geodesy, edited by G. Schubert, pp. 391-446, Elsevier, Amsterdam.

Tichelaar, B. W., and L. J. Ruff (1989), How good are our best models?, Eos Trans. AGU, 70, 593-605.

Zebker, H. A., P. Rosen, and S. Hensley (1997), Atmospheric effects in interferometric synthetic aperture radar surface deformation and topographic maps, J. Geophys. Res., 102, 7547-7563.

Zhong, S., J. Li, C. D. Whiteman, X. Bian, and W. Yao (2008), Climatology of high wind events in the Owens Valley, California, Mon. Weather Rev., 136(9), 3536-3552, doi:10.1175 2008MWR2348.1. 